\title{
Oral Hygiene Perceptions and Practices among Population of Al-Qassim Region, KSA
}

\author{
Ahmed Obied Almahrul ${ }^{1, *}$, Ahmed Mohammed Aldakhil ${ }^{2}$ \\ ${ }^{1}$ King Abdulaziz University Dental Hospital, King Abdulaziz University, Jeddah, KSA \\ ${ }^{2}$ College of Dentistry, Qassim University, Buraidah, KSA \\ *Corresponding author: Ahmed.aldakhil@qudent.com
}

Received August 04, 2018; Revised September 06, 2018; Accepted September 17, 2018

\begin{abstract}
Oral health knowledge is considered to be an essential prerequisite for health related behavior. Studies have shown that there is an association between knowledge and better oral health. Although many studies have been carried out from time to time to assess the knowledge and behavior of people about oral health, there is still a dearth of education regarding the same especially for rural people in Saudi Arabia. The aim of the present study is to identify the daily performed oral hygiene practices and awareness of Saudi dental patients towards oral hygiene maintenance and to assist Saudi dental authorities to provide and enhance the governmental dental services. After obtaining the Institutional Ethical Committee approval, 199 Saudi nationals (male \& female) visiting the dental clinics, College of dentistry, Qassim University were selected randomly and questionnaires were handed over for the needed information. The data was statistically evaluated using Chi square test. $\mathrm{p}$ value of 0.05 will be considered as level of significance. The results showed statistically significant results with respect to the parameters such as methods and frequency of tooth brushing, responses related to use of other dental cleaning Aids, Awareness for oralsystemic health relations, frequency and cause of visiting dentist and awareness about bleeding gums \& oral Malodor. It is concluded that even though there is fair knowledge about the oral health awareness among the people, more awareness projects are needed by the higher Saudi authorities in implementing and following them meticulously for better overall oral and general health.
\end{abstract}

Keywords: awareness, knowledge, practice, oral hygiene, systemic health, oral hygiene recommendations, Al-Qassim; KSA

Cite This Article: Ahmed Obied Almahrul, and Ahmed Mohammed Aldakhil, "Oral Hygiene Perceptions and Practices among Population of Al-Qassim Region, KSA.” International Journal of Dental Sciences and Research, vol. 6, no. 5 (2018): 129-133. doi: 10.12691/ijdsr-6-5-4.

\section{Introduction}

Oral hygiene is to no surprise the core of preventive dentistry that must be emphasized upon and ensure that it is considered seriously by both the dental professional and dental service seeker. When given its rightfully earned place in the field of dentistry, oral hygiene education and emphasizing of oral health practices can and will be extremely helpful in changing the dental professional service both in the level of required services and the level of noticed results.

It is a common sense that keeping a fairly good oral hygiene would render the mouth less susceptible to developing dental or oral infections. Moreover, simple daily dental hygiene practices save much efforts and money as well that would be spent just to restore one's oral health status to normal.

Out a survey titled "Oral Hygiene Practice among Saudi patients in Jeddah" [1] the objective of which was to investigate the oral self-care habits (frequency of tooth brushing and flossing) and the attitude towards preventive dental visits. The conclusion reached by the surveyor was that professional plaque removal and regular follow-up combined with patient oral hygiene instructions can minimize the level of gingival inflammation and swelling. So, the lack of patient compliance in home care and regular dental visit can therefore be seen as the key problem in the prevention of periodontal disease [1].

Another study conducted by Al-Kheraif and Al-Bejadi titled "Oral Hygiene awareness among female Saudi school children" evaluated the oral hygiene habits and utilization of professional dental health services by all the children in the elementary schools, and to compare the differences in oral hygiene awareness and dental health status of school children who are exposed to dental health education and those who are not. [2]

It involved 400 Saudi school girls chosen in a random fashion from female elementary schools in the city of AlKharj, then divided into two equal groups of 200 girls; a study group of girls who were exposed to dental health education program and a control group of girls who were not exposed to the program. The results revealed that brushing twice a day was more common in the study group than the control group. However, no significant 
difference was observed between both groups regarding use of dental floss, mouthwash or routine checkup visit to dentist. [2]

The information on developments in vital combination of Oral Hygiene, Oral Diagnosis, and overall health needs to be spread by us, the dentists. Establishing and demonstrating this connection will be critical to achieve this goal and this process will have to be taken at all levels including a definite beginning with our patients. All of these and more innovative methods of reaching the public will not only ensure a healthy individual but a healthy society as well. [3] Furthermore, a fourth study was made to assess the frequency of consumption of cariogenic foods, oral hygiene practices and dental health knowledge among Saudi male primary school children in relation to socio-demographics and to find the possible predictors for dental caries among them. It included 1115 Saudi males, who were selected randomly. These samples were interviewed using close ended questionnaire pertaining to frequency of consumption of some cariogenic foods, oral hygiene practices and dental health knowledge. They underwent dental screening to detect the clinically evident carious lesions. The results showed that the clinically decayed tooth was diagnosed in $68.9 \%$ of the included children, more in urban and younger students. Caries affected the subjects who consumed cariogenic foods at greater frequency compared with caries-free children. Only $24.5 \%$ of the students brushed their teeth twice or more per day, and $29 \%$ of them never received instructions regarding oral hygiene practices. [4]

\section{Material and Method}

\subsection{Study Objectives}

A self-administered questionnaire was developed to identify the daily performed oral hygiene practices and awareness of Al-Qassim Region population in KSA as well as relate systemic-oral health and compare oral hygiene perceptions and practices between male and female.

\subsection{Study Design and Sample}

Cross sectional study was performed. A questionnaire based study was carried out. The questionnaire was designed in Arabic language. The sample consisted of 199 (male 99\& female 100) randomly selected from Saudi dental resident patients visiting dental clinics in faculty of dentistry, Qassim university, governmental hospitals and primary health care centers in Al-Qassim region, KSA. Inclusion criteria were Saudi patients visiting dental clinics for dental problems with age range 18 years and older. In addition, patients with abnormalities and syndromes.

\subsection{Ethical Considerations}

This study was approved by the Dental Ethics Committee and Dental Students' Research Facilitation Committee in Qassim University, College of Dentistry.

\subsection{Data Analysis}

Data were coded and keyed into the Statistical Package for the Social Sciences software version 21 (SPSS Inc., Chicago, IL) for analysis and to perform Pearson Chi-square test for statistical significance ( $\mathrm{P}$ value). The $\mathrm{P}$ value less than 0.05 was considered statistically significant.

\section{Results}

In this study a total of a total of 199 patients (99 male \&100 female) randomly selected visiting the dental clinics, with age group range 12-65 years with average of 20-35 years. The interpretation of results for the included parameters is as follows (Table 1).

An average of $60 \%$ of participants (58.6\% males \& $62 \%$ females) were brushing teeth as a regular oral hygiene maintenance measures which is statistically not significant $(\mathrm{p}=0.623)$. However, the majority of males (53.5\%) brushed once a day, about $19.2 \%$ brushed thrice daily and only $14.1 \%$ brushed twice a day. To compare with females $52.5 \%$ brushed three times a day, $24.2 \%$ twice a day and about $13.1 \%$ once a day. The statistical analysis was highly significant for the frequency of brushing.

Table 1. Distribution of the Study Population by Oral Hygiene Practices and Gender

\begin{tabular}{|c|c|c|c|c|}
\hline \multirow{2}{*}{\multicolumn{2}{|c|}{ The Question and The Answer }} & \multicolumn{3}{|c|}{ Gender } \\
\hline & & \multirow{2}{*}{$\begin{array}{c}\text { Male \% } \\
58.6 \%\end{array}$} & \multirow{2}{*}{$\begin{array}{c}\text { Female } \% \\
62.0 \%\end{array}$} & \multirow{3}{*}{$\begin{array}{c}\text { Sig. } \\
0.623\end{array}$} \\
\hline \multirow{2}{*}{ Teeth Brushing } & Yes & & & \\
\hline & No & $41.4 \%$ & $38.0 \%$ & \\
\hline \multirow{5}{*}{$\begin{array}{l}\text { Other Dental } \\
\text { cleaning Aids }\end{array}$} & Mouth Wash & $63.6 \%$ & $31.0 \%$ & \multirow{5}{*}{0.001} \\
\hline & Tooth Picks & $27.3 \%$ & $31.0 \%$ & \\
\hline & Superfloss & $8.1 \%$ & $36.0 \%$ & \\
\hline & Interdental Brush & $1.0 \%$ & $1.0 \%$ & \\
\hline & Other & $0.0 \%$ & $1.0 \%$ & \\
\hline \multirow{4}{*}{$\begin{array}{c}\text { Frequency of } \\
\text { Visiting Dentist } \\
\text { for Scaling }\end{array}$} & Not Usually & $58.6 \%$ & $26.0 \%$ & \multirow{4}{*}{0.001} \\
\hline & Every 3 Month & $17.2 \%$ & $29.0 \%$ & \\
\hline & Every 6 Month & $18.2 \%$ & $34.0 \%$ & \\
\hline & Every 1 Year & $6.1 \%$ & $11.0 \%$ & \\
\hline \multirow{2}{*}{$\begin{array}{l}\text { Is this First } \\
\text { Scaling Visit }\end{array}$} & Yes & $61.6 \%$ & $30.0 \%$ & \multirow{2}{*}{0.001} \\
\hline & No & $38.4 \%$ & $70.0 \%$ & \\
\hline \multirow{4}{*}{$\begin{array}{c}\text { Previous Scaling } \\
\text { Visits }\end{array}$} & Less Than 5 & $52.9 \%$ & $32.4 \%$ & \multirow{4}{*}{0.173} \\
\hline & Less Than 10 & $23.5 \%$ & $42.3 \%$ & \\
\hline & Less Than 20 & $14.7 \%$ & $18.3 \%$ & \\
\hline & Less Than 20 & $8.8 \%$ & $7.0 \%$ & \\
\hline \multirow{4}{*}{$\begin{array}{l}\text { Cause to Clean } \\
\text { Teeth Today }\end{array}$} & Dentist's Request & $31.3 \%$ & $23.0 \%$ & \multirow{4}{*}{0.001} \\
\hline & Gum Problem & $47.5 \%$ & $27.0 \%$ & \\
\hline & Regular Visit & $19.2 \%$ & $50.0 \%$ & \\
\hline & Others & $2.0 \%$ & $0.0 \%$ & \\
\hline \multirow{2}{*}{$\begin{array}{c}\text { Relation between } \\
\text { Oral and } \\
\text { Systemic Health }\end{array}$} & Yes & $41.4 \%$ & $58.0 \%$ & \multirow{2}{*}{0.019} \\
\hline & No & $58.6 \%$ & $42.0 \%$ & \\
\hline \multirow{4}{*}{$\begin{array}{l}\text { Types of } \\
\text { Systemic } \\
\text { Diseases }\end{array}$} & CVS Diseases & $35.0 \%$ & $15.5 \%$ & \multirow{4}{*}{0.041} \\
\hline & $\mathrm{Dm}$ & $50.0 \%$ & $50.0 \%$ & \\
\hline & Bact. Pneumonia & $15.0 \%$ & $27.6 \%$ & \\
\hline & Lbw & $0.0 \%$ & $6.9 \%$ & \\
\hline \multirow{2}{*}{ Bleeding Gums } & Yes & $62.6 \%$ & $53.0 \%$ & \multirow{2}{*}{0.169} \\
\hline & No & $37.4 \%$ & $47.0 \%$ & \\
\hline \multirow{2}{*}{ Bad Odor } & Yes & $77.8 \%$ & $86.0 \%$ & \multirow{2}{*}{0.13} \\
\hline & No & $22.2 \%$ & $14.0 \%$ & \\
\hline
\end{tabular}



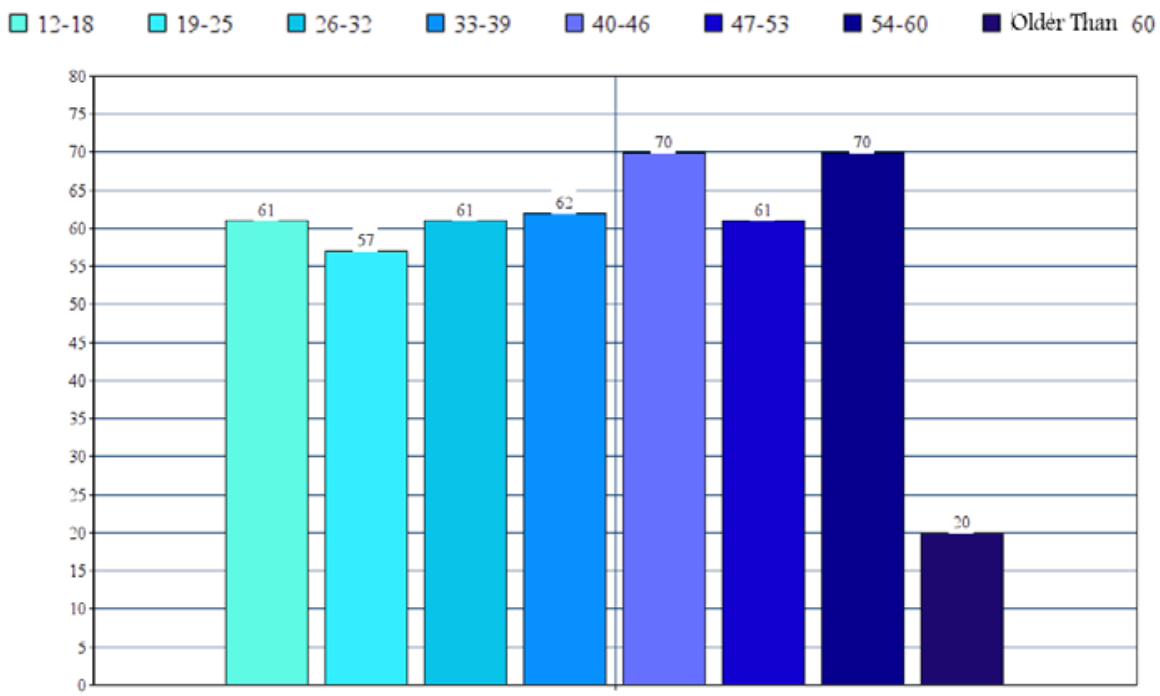

Teeth Brushing

Figure 1. Percentage of Population Aged 12 and Over that Confirmed they Brushing their Teeth at Least One a Day



Figure 2. Comparison between Male and Female Regarding the Frequency of Teeth Brushing

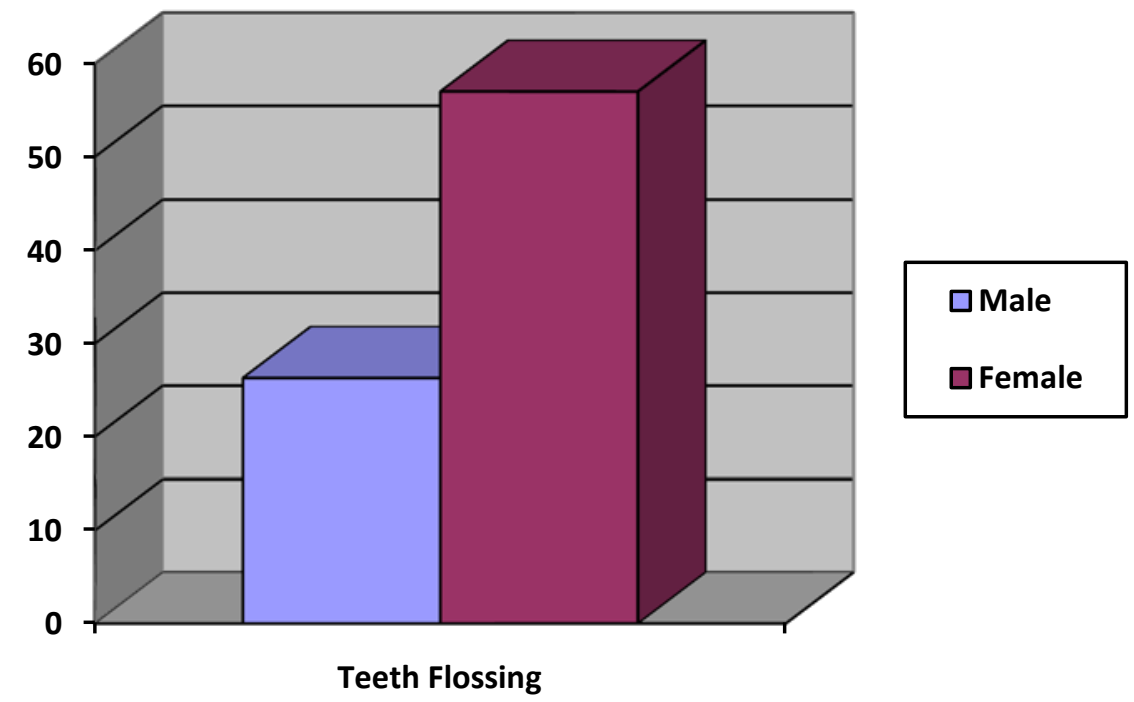

Figure 3. Comparison between Male and Female Regarding the Frequency of Teeth Flossing 


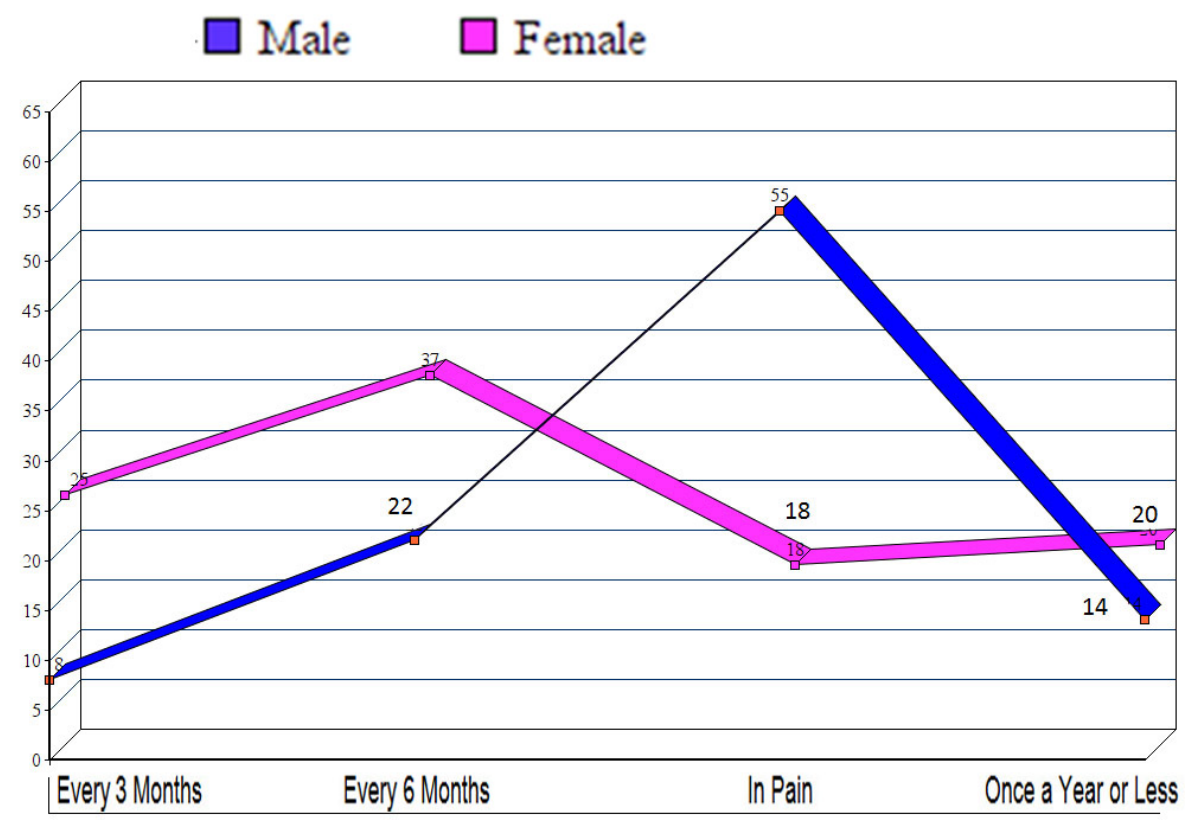

Figure 4. Comparison between Male and Female Regarding the Frequency of of Visiting the Dental Clinic.

The oral hygiene practices about $37 \%$ of the included patients stated use of teeth flossing, of which 52\% (35.7\% males \& 70.5\% females) flossed three times a day and $15 \%$ once ad day. The results were statistically significant different between male and female with teeth flossing in $\mathrm{P}$ value $(\mathrm{P}<0.05)$

Responses of dental health knowledge revealed that Mouthwash (50\%), tooth picks (29\%) and super-floss (20\%) were the other dental cleaning aids used with females showing more inclination towards the use of super-floss(36\%). The results were statistically highly significant $(\mathrm{p}=0.0001)$ indicating a good awareness among the patients for the use of other dental cleaning aids in oral hygiene maintenance.

The need of visiting the dental clinic showed that majority of male patients visited when in pain(55\%) and $22 \%$ visited every 6 months as routine dental checkups with frequency of visiting dentist for scaling is about $42 \%$. Where, among the females the majority visited the dentist as routine dental check up 3-6 months (65\%) and usually for the scaling (74\%) showing higher awareness towards the oral hygiene maintenance.

Results showed that of the new patients, about $61 \%$ of males were their first scaling visit about half of them wanted scaling for their gum problems (47\%) showing higher awareness for their periodontal health. However, female statistics showed that only $30 \%$ were new patients.

Responses for knowledge of relation between oral health to systemic heath revealed $50 \%$ were aware of the inter-relation and majority of them were knew about diabetes mellitus(50\%), and other systemic disease (CVS diseases 26\% \& Bact. Pneumonia 24\%) with statistical significance $(\mathrm{p}=0.041)$.

\section{Discussion}

The aim of the present study was to identify the daily performed oral hygiene practices and awareness of Al-Qassim Region population, KSA towards oral hygiene maintenance. Our study shows that there is fair awareness among the patients for oral health with $40 \%$ of them utilizing the dental services as routine dental check-up. Additionally, the majority visits only in case of pain. Preventive oral health education is in transitional stage in Saudi Arabia. Still population based oral health promotional programs are yet to be implemented and followed on regular basis.

The relationship between dental service utilization and main demographic variables (sex. Education) are discussed in this study. Our study shows that female patients have more awareness towards utilization of dental services as well as more dental cleaning aids than the male patients, which supports the reports of Helsinki et al [6].

Our study reports that $60 \%$ of patients use Brushing as the most common method of teeth brushing. The reports also showed fair percentage (37\%) of patients uses other dental cleaning aids such as super-floss, tooth picks and mouth washes for their regular oral hygiene maintenance. These reports are in contrast with the results of Jamjoom HM [7] conducted in Saudi Arabia in 2001, where no subject used dental floss for interdental cleaning. These results show low awareness among Al-Qassim Region population regarding use of modern dental cleaning aids.

Visiting a dentist is still not considered a preventive dental behavior, at present it only depends on the treatment needs [8]. The present study shows that around $55 \%$ of the patients visited the dentist only in problem and only $22 \%$ of the population visited the dentist on regular basis after every 6 months. These results are similar to the study done by Jain et al. where $54 \%$ of the subjects visited the dentists when they were in pain [3].

\section{Conclusions}

The present study emphasizes on the awareness of Saudi patients towards oral health maintenance habits and as well about systemic-oral health relationship. Our study has shown less than expected knowledge about oral health 
and systemic health inter-relationship among Al-Qassim Region population. Furthermore, the higher authorities should take up awareness projects to implement. In addition, the focus should be on the most vulnerable groups in society, such as children and adults with disabilities.

The positive results were promising as compared to the results of previous studies. It is concluded that even though there is fair knowledge about the oral health awareness among the Al-Qassim Region population, more awareness projects are needed by the higher Saudi authorities in implementing and following them meticulously for better overall oral and general health. In fact, more studies should be conducted at multicenter level for a larger population for more accurate results.

\section{Acknowledgements}

I would like to express my sincere gratitude to Dr.Dhafer Al Asmari the Dean of college of dentistry, Qassim University, KSA for the continuous support. Also, I would like to thank the Department of Dental Services, Ministry of Health, Al-Qassim Region, KSA. for allowing the author to distribute and collect the data.

\section{References}

[1] Hind Al-Johani, (2008). Oral Hygiene Practice among Saudi Dental Patients in Jeddah; Cairo Dental Journal, 24 (3): 395-401.

[2] Abdulaziz A. Al-Kheraif, Shaikhah A. Al-Bejadi, (2008). Oral hygiene awareness among female Saudi school children. Saudi Medical Journal; 29 (9): 1332-1336.

[3] Nitika Jain, Dipika Mitra, K. P. Ashok, Jyothi Dundappa,et al (2012). Oral hygiene-awareness and practice among patients attending OPD at Vyas Dental College and Hospital. 16(4): 524-528.

[4] Amin T., Al-Abad B.(2008) Oral hygiene practices, dental knowledge, dietary habits and their relation to caries among male primary school children in Al Hassa, Saudi Arabia. International Journal of Dental Hygiene. 6(4): 361-370.

[5] Daljit Kapoor, Sanjeet Gill, Arshdeep Singh, et al (2014). Oral hygiene awareness and practice amongst patients visiting the Department of Periodontology at a Dental College and Hospital in North India. 5(2): 64-68.

[6] Ventä I, Murtomaa H, Meurman J, Turtola L, (1992) Use of dental services by students of Helsinki University during six years. Proc Finn Dent Soc.; 88(3-4): 123-9.

[7] Jamjoom HM (2001). Preventive Oral Health Knowledge and Practice in Jeddah, Saudi Arabia. J KAU Med Sci. 9: 17-25.

[8] Gundala R, Chava VK (2010). Effect of lifestyle, education and socioeconomic status on periodontal health. Contemp Clin Dent. 1 23-6. 